\title{
Identification of the Specification Parameters for a Voltage Controlled Oscillator Using an Artificial Neural Network with a Genetic Algorithm
}

\author{
Sebastian Temich, Lukas Chruszczyk, Damian Grzechca \\ Faculty of Automatic Control, Electronics and Computer Science, \\ Institute of Electronics, Silesian University of Technology, \\ Akademicka 16, 44-100, Gliwice, Poland \\ damian.grzechca@polsl.pl
}

\begin{abstract}
This paper presents the application of an artificial neural network with a genetic algorithm for identifying the selected specification parameters of a voltagecontrolled oscillator (VCO). In modern electronics, the complexity of the production process may cause errors in analogue and mixed-signal electronic circuits, and inaccuracies in this technological process have a direct impact on the specification parameters of a VCO. The modern market requires that the production process has to be as quick as possible, and therefore testing systems should be fast and have the highest efficiency of parameter identification. In the following paper, a genetic algorithm is used to optimise the number of output signal measurement points, which allows them to be identified by the specification parameters of the VCO that are selected by an artificial neural network. The proposed method is characterised by shortening the test time of the system while maintaining a high efficiency in the identification of the selected design specification parameters.
\end{abstract}

Index Terms-Voltage-controlled oscillator; Specification parameters; Identification; Genetic algorithm; Optimisation; Artificial neural network.

\section{INTRODUCTION}

Fault diagnosis of analogue circuits is an important and still relevant element for the design validation of electronic devices [1]-[5]. The problem of diagnosis is complex due to the large number of process variables, which can be altered during production.

Fault diagnosis can be divided into two main sections: it includes detecting faulty circuits, locating faulty parameters and evaluating their values and determining whether a faulty parameter has drifted from its tolerance range but does not lead to any topological changes [6].

Analogue circuit testing can be divided into two categories: Fault Driven Testing (FDT), which detects faults in elements or Functional Test/Specification Driven Test (FT/SDT), where the functional behaviour of the circuit being tested is measured. In this case, the test should be

Manuscript received 26 April, 2018; accepted 7 September, 2018.

This work was supported by the Polish Ministry of Science and High Education funding for statutory activities. designed to gather all of the possible datasheet information. However, due to the easy availability of electronic circuits and the low cost of their execution, the testing of systems is aimed at checking the design specifications in order to check their compliance with the adopted assumptions [7].

In order to speed up the launch of a product to the market, the Simulation Before Test stage methodology (SBT) has been introduced. In the SBT approach, a testing procedure is created and a circuit under test (CUT) numerical implementation is used. All of the possible parametric deviations are simulated. The desired specification parameters of CUT are calculated for all of the simulations and a fault dictionary is created. On the production line, a manufactured circuit is tested using the same procedure and the CUT response is compared with the fault dictionary [7].

Nowadays, among the multiplicity of the test algorithms, most attention has been focused on the heuristic methods, evolutionary techniques, fuzzy logic, support vector machines and artificial neural networks [1]-[9].

While most of the works have been focused on the soft fault diagnosis of analogue circuits when only one parameter is faulty, fewer papers have been directed to multiple faults [8], [10]-[18]. This paper presents the use of an artificial neural network (ANN) to reduce the identification time of Voltage-Controlled Oscillator selected specification parameters. In the identification procedure (Fig. 1), the output signal samples of the generator are selected by the optimisation of the genetic algorithm (GA). The selected samples become the input for the artificial neural network. Based on the selected samples, the learned ANN determines the value of the selected specification parameters of the circuit under test (CUT).

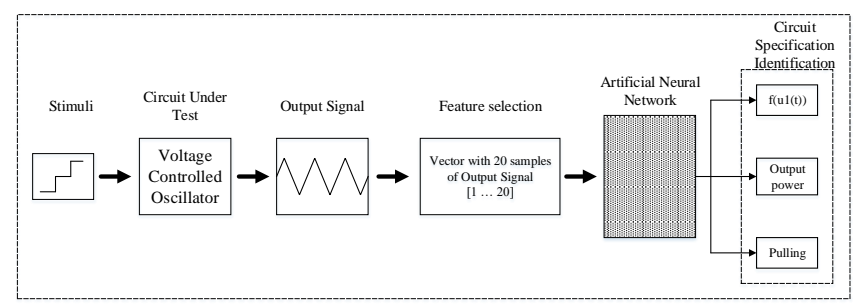

Fig. 1. Testing idea. 
The paper is organised as follows. In Section II, a model of a CUT-Voltage Controlled Oscillator is presented and the specification parameters are introduced. Section II also raises the issue of the imperfectness of production, where the parametric faults of CUT are described. Sections III and IV are devoted to the description of the genetic algorithm (Sec. III) and the artificial neural network (Sec. IV). Finally, the last section describes the results of the research in detail.

\section{II.CIRCUIT TESTING}

\section{A. A Voltage-Controlled Oscillator}

Oscillator topologies are classified as tuned, ring and crystal oscillators. Tuned oscillators are implemented as LC or RC circuits. Ring oscillators are basically delay stages that are connected by positive feedback, which are easy to implement because of the simple integration of CMOS technology. LC oscillators occupy more space than their ring counterparts [19]-[21].

The circuit under test is a voltage-controlled oscillator (VCO) based on a ring oscillator topology. In the classical structure, a VCO contains the control input stage, the ring oscillator and the output buffer. The five-stage ring oscillator topology, which is based on NMOS and PMOS transistors, is presented in the Fig. 2.

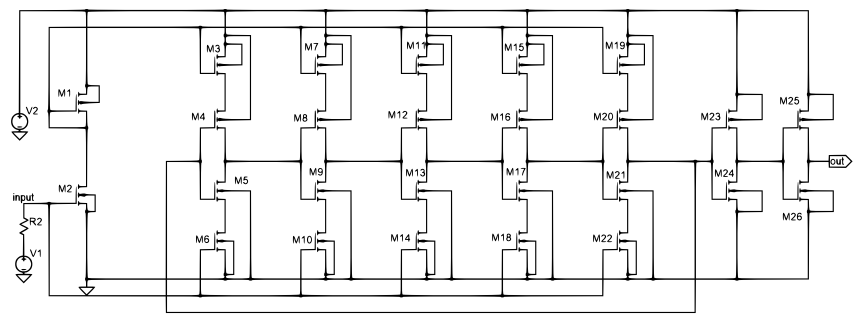

Fig. 2. Five-stage voltage-controlled oscillator.

The purpose of a voltage-controlled oscillator (VCO) is to generate an output signal $\left(\mathrm{u}_{\mathrm{out}}^{\mathrm{i}}\right)$ with an oscillation frequency that is proportional to the control voltage signal $\left(u_{i n}^{i}\right)$ [22]. Transistors NMOS M4 and PMOS M5 (Fig. 2) operate as inverters and create one structure of the oscillator. The inverter structure is repeated five times creating a fivestage ring oscillator.

\section{B. Voltage-Controlled Oscillator Functional Parameters}

The functional identification of a circuit under test should be focused on measuring the different characteristics of the output signal for the stimuli being tested. The selected functional parameters affect the quality of the voltagecontrolled oscillator that is produced. According to the parametric errors of a VCO structure, a functional test should contain:

- a frequency $f x\left(u_{i n}^{i}\right)$ response for the input signal $\left(u_{i n}^{i}\right)$ [6],

- a frequency tuning characteristic $(f x(V))$, which expresses the relationship between the VCO operating frequency at the tuning voltage that is applied [21],

- output power $\left(P_{d B m}\right)$, which is measured into a $50 \Omega$ load [21],

- Pulling (Pull in $\mathrm{dBr}$ ), which describes the frequency variation in any change of the output load [21],

- phase noise $\left(P_{N}\right.$ in $\left.\mathrm{dBc} / \mathrm{Hz}\right)$, which describes the single sideband phase noise of the oscillator. It is composed of the noise close to the flicker noise and the noise measured at a spacing of a constant value [21],

- VCO gain $\left(K_{V C O}\right)$, which is measured in $(\mathrm{Hz} / \mathrm{V})$, which describes any change in the frequency value due to a change in the input signal voltage [21],

- frequency tuning range $\left(f x_{R}\right)$, which is the difference between the maximum and minimum output signal frequency values [21].

As was mentioned above. voltage-controlled oscillator circuits are characterised by a frequency response, and therefore, it is important that the frequency of the designed generator for the applied voltage is within the desired range, therefore the circuit testing process starts with a frequency measurement $\left(f x\left(u_{i n}^{i}\right)\right)$ for the testing voltage $\operatorname{signal}\left(u_{i n}^{i}\right)$.

Because the output of an oscillator is always attached to some load, which can affect the frequency of the VCO, it is also important to measure the output signal frequency as a function of the changing of the load of the CUT - Pulling of the VCO $\left(\right.$ Pull $\left.^{i}\right)$.

Finally, a voltage-controlled oscillator is also characterised by an output power $\left(P_{d B m}^{i}\right)$ specification parameter.

This paper is focused on identifying the $f x\left(u_{i n}^{i}\right)$, $P_{d B m}^{i}$ and $P u l l^{i}$ for each transistor parametric fault. The nominal values of $f x\left(u_{i n}^{i}\right), P_{d B m}^{i}$ and $P u l l^{i}$ for the nominal transistor parameter are presented in Table I.

TABLE I. NOMINAL VALUES OF THE VCO SPECIFICATION PARAMETERS.

\begin{tabular}{|c|c|c|c|}
\hline & $\boldsymbol{f} \boldsymbol{x}\left(\boldsymbol{u}_{\boldsymbol{i n}}^{\boldsymbol{i}}\right)[\mathbf{G H z}]$ & $\boldsymbol{P}_{\boldsymbol{d B m}}^{\boldsymbol{i}}[\mathbf{d B m}]$ & $\boldsymbol{P u l l}^{\boldsymbol{i}}[\mathrm{dBr}]$ \\
\hline $\boldsymbol{u}_{\boldsymbol{i n}}^{\mathbf{1}}$ & 1.381 & 1.4003 & -96.9 \\
\hline $\boldsymbol{u}_{\boldsymbol{i n}}^{\mathbf{2}}$ & 0.387 & 1.447 & -110.5 \\
\hline $\boldsymbol{u}_{\boldsymbol{i n}}^{\mathbf{3}}$ & 2.583 & 1.463 & -93.9 \\
\hline $\boldsymbol{u}_{\boldsymbol{i n}}^{\mathbf{4}}$ & 3.009 & 1.487 & -107.9 \\
\hline
\end{tabular}

\section{Production Imperfectness}

As can be seen in the Fig. 2, the VCO presented in this paper is based on PMOS and NMOS transistors. Transistors can be parametrically damaged in many different ways during technological processes. The most frequent parametric damage at the production stage is a deviation of the photolithographic mask [23]-[24]. This kind of error affects the parametric values of the transistor structure - the width $(\mathrm{W})$ and length $(\mathrm{L})$ of a channel. A change to this parameter in turn has a significant impact on the transistor specification parameters (i.e. the amplification of the transistor), which has an effect on the specification parameters of the voltage-controlled oscillator.

During computer analysis, the VCO is modelled using Spice software and the transistor fabrication process is modelled by width (W) and length (L).

The nominal values of $\mathrm{W}$ and $\mathrm{L}$ in NMOS (N) and PMOS (P) are defined $W_{n o m}^{N}, L_{n o m}^{N}, W_{n o m}^{P}, L_{n o m}^{P}$. The maximum range for the $\mathrm{W}$ and $\mathrm{L}$ parameter was set to: 
$\left\{\begin{array}{l}W^{N / P} \in<W_{\min }^{N / P} ; W_{n o m}^{N / P}>, W^{N / P} \in<W_{n o m}^{N / P} ; W_{\max }^{N / P}>, \\ L^{N / P} \in<L_{\min }^{N / P} ; \quad L_{n o m}^{N / P}>, L^{N / P} \in<L_{n o m}^{N / P} ; \quad L_{\max }^{N / P}>.\end{array}\right.$

The transistor nominal range is represented by:

$$
\left\{\begin{array}{l}
W_{\text {nom }}^{N / P} \in<W_{\text {nom }}^{N / P}-t_{W}^{N / P} ; W_{\text {nom }}^{N / P}+t_{W}^{N / P}> \\
L_{\text {nom }}^{N / P} \in<L_{\text {nom }}^{N / P}-t_{L}^{N / P} ; \quad L_{\text {nom }}^{N / P}+t_{L}^{N / P}>
\end{array}\right.
$$

where $t_{W}^{N}, t_{W}^{P}, t_{L}^{N}, t_{L}^{P}$ are the tolerance ranges.

To analyse a large number of photolithographic mask deviations, a number of Monte Carlo analyses with uniform distributions were created ( $M_{C}$ runs).

To increase the accuracy of the analysis of the CUT, the parametric damage to the NMOS and PMOS was divided into $Y$ subranges. Let $Y$ represent the width or length and $Y^{\text {nom }}$ be the nominal values, the new subrange middle value $Y^{\text {mid }}$ is described as

$$
Y_{i}^{m i d}=Y_{i}^{\text {nom }} \times t_{i}
$$

where $t_{i} \in\{0.88,0.94,1,1.06,1.12,1.18\}$.

Using the above, each $Y^{\text {mid }}$ is defined

$$
Y_{i}^{m i d} \in<Y_{i}^{\text {nom }} \times t_{i}-t_{L}^{N / P} ; Y_{i}^{n o m} \times t_{i}+t_{L}^{N / P}
$$

\section{Simulation Profile}

The aim of the proposed method presented in this paper is to identify the selected specification parameters of a VCO in case of NMOS and PMOS transistor parametric faults. For the transistors from Fig. 2, the nominal parameter represents $50 \mathrm{~nm}$ and was set to [24]:

$$
\left\{\begin{array}{l}
W_{n o m}^{N}=0.5 u, \\
L_{n o m}^{N}=50 n, \\
W_{n o m}^{P}=1 u, \\
L_{n o m}^{P}=50 n .
\end{array}\right.
$$

$M_{c}=500$ Monte Carlo analysis was generated for each simulated set, and the tolerances for each simulation was set as

$$
t_{W}^{N}=t_{W}^{P}=t_{L}^{N}=t_{L}^{P}=3 \%
$$

At the simulation stage, four-step functions were considered: $u_{i n}^{1}=u_{i n}^{1} \cdot 1(t), u_{i n}^{2}=u_{i n}^{2} \cdot 1(t), u_{i n}^{3}=u_{i n}^{3}$. $1(t)$ and $u_{i n}^{4}=u_{i n}^{4} \cdot 1(t)$, where $u_{i n}^{1}=0.8 \mathrm{~V}, u_{i n}^{2}=1 \mathrm{~V}$, $u_{i n}^{3}=1.2 \mathrm{~V}, u_{i n}^{4}=1.5 \mathrm{~V}$, for all $u_{i n}$, the rising time $\left(\right.$ time $\left._{r}\right)$ was equal - time $_{r}=0.1 \mathrm{~ns}$. The exemplary response for transistors $Y_{3}^{\text {mid }}$ (continuous line) and $Y_{4}^{\text {mid }}$ (dotted line) is presented in Fig. 3.

\section{GENETIC ALGORITHM}

In order to solve a given optimisation problem, a genetic algorithm (GA) was used. The GA has proven to be robust and acceptably quick for problems of multi-parameter optimisation [24].

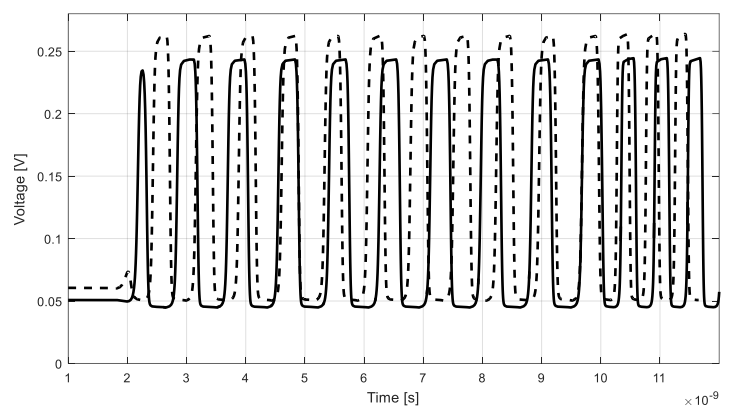

Fig. 3. Circuit response for $Y_{4}^{\text {mid }}$ (continuous line) and $Y_{5}^{\text {mid }}$ (dotted line).

However, as with all of the heuristic methods, there is no formal proof of its global convergence, and therefore no guarantee that solution that is found is definitely the optimal one [25] The classic elitist GA with the schema shown in the (Fig. 4) was used [25].

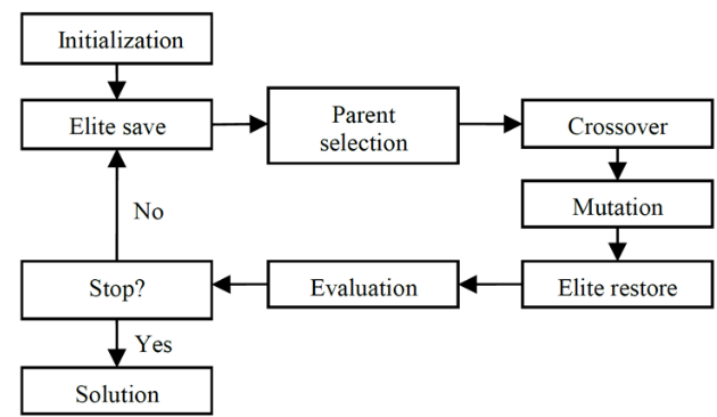

Fig. 4. Block diagram of the genetic algorithm (an elitist model) that was used [21].

In the proposed $\mathrm{GA}$, the population size $\left(\mathrm{P}_{\mathrm{S}}\right)$ is constant. Each individual contains a single linear chromosome, which is a vector of genes $\left(\mathrm{V}_{\mathrm{g}}\right)$ that is coded as bits and represents a particular solution. The length of the chromosome is a trade-off between the accuracy of the solution (bit resolution) and the processing time of the genetic algorithm.

The evolution loop is built from the following steps (Fig. 4):

1. Initialization - using random values,

2. elite individuals, where the unique individuals are saved. This step prevents the best solutions that have been found so far from being destroyed in the next genetic operations (i.e. crossover),

3. parent selection, which is based on a binary tournament. Two individuals are randomly selected (with return) from the main population. The one with the lower (better) value of fitness is copied to the population of parents,

4. reproduction (single-point crossover) is applied to the randomly selected pairs of parents and the offspring population is created,

5. the succession population of offspring replaces the old main population,

6. mutation, the negation of each gene using probability,

7. the elite is restored into the main population (randomly selected individuals are replaced in order to maintain a constant population size). A random overwrite does not introduce any evolutionary pressure,

8. the new population is evaluated and the fitness of an individual is calculated,

9. stop criterion - GA stops and returns the solution that was found after a specified number of iterations. 
The proposed genetic algorithm was used to find the global minima of each of the selected specification parameters. The GA algorithm was created with the following parameters:

1. initial population as being uniformly random,

2. selection operator as a binary tournament with repetition,

3. crossover as a single-point,

4. succession: complete,

5. mutation as a uniform with a mutation probability for a single gene set to 0.01 ,

6. elite saving as one or more individuals with the best fitness and a unique genotype,

7. $P_{\mathrm{S}}$ was set to 100 individuals,

8. size of the parent and offspring populations is equal in size to the main population,

9. stop criterion where the maximal number of generations (iterations) is equal 50.

The main optimisation goal is to reduce the testing time. This can be done by reducing the number of measurement samples (Sample) and shortening the length of the vectors of the measurement samples $\left(S_{u_{\text {out }}}\right)$. Each $S_{u_{\text {out }}}$ is created by $s$ selected indexes $w$ from the vector Sample. In order to shorten the chromosome, a seven-bit resolution of binary vector $B$ was selected. Then, every second index $w$ from the vector Sample could be selected, which is sufficient. Therefore, each $w$ is calculated as

$$
w=2 \times b+1,
$$

where $b=\operatorname{graydecode}(B)$ and $b \in\{0,1,2, \ldots, 127\}$ and $w \in\{1,3, \ldots, 255\}$.

Each binary vector $B$ encodes a single index $w$. The chromosome length is calculated as $S_{u_{\text {out }}} \cdot B$.

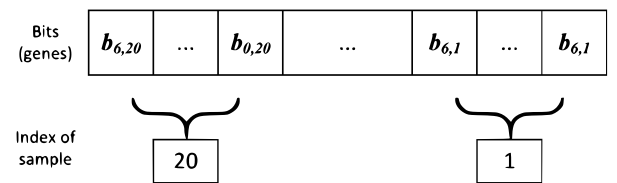

Fig. 5. Chromosome coding indices of the samples using seven bits (genes).

The progress of the genetic algorithm that was run is presented in (Fig. 6).

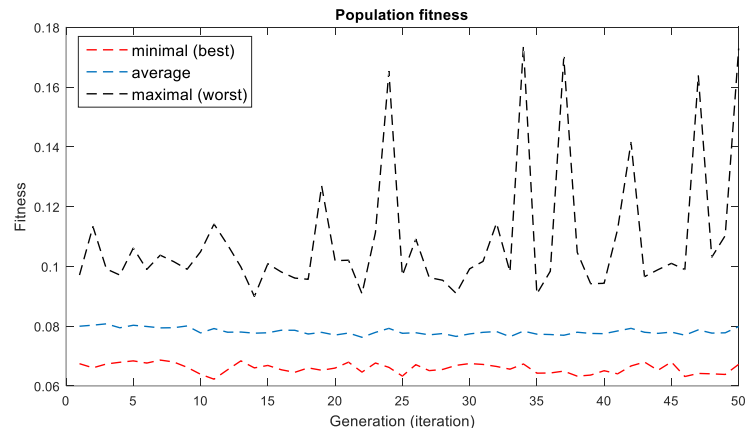

Fig. 6. Fitness progress of GA evolution.

The average fitness of the population decreased, which is the desirable behaviour. The individuals that represented bad solutions, where high performance values $\equiv$ low fitness, died during the evolution process. The individuals that represented good solutions, where low performance values $\equiv$ high fitness had a greater survival probability, reproduced and propagated in the population.

The generalization ability of the ANN overlap the GA elitism can be seen, e.g. in the Fig. 6, Fig. 15. Therefore, the GA progress was not significant. In such a case, the application of elitism in the GA algorithm is of a weaker importance. However, the ANN performance can even be different for exactly the same inputs (measurement samples) and the GA may work better for a different fitness function.

\section{ARTIFICIAL NEURAL NETWORK}

In the presented paper, an artificial neural network (ANN) with the genetic algorithm was used to identify the selected VCO specification parameters frequency $\left(f x\left(u_{i n}^{i}\right)\right)$, output power $\left(P_{d B m}^{i}\right)$ and pulling $\left(P u l l^{i}\right)$ for selected stimuli). The presented ANN is described as follows

$$
A N N=\{\text { in }, l, o u t\}
$$

where in represents the ANN inputs and out represents the ANN outputs. The $l$ variable is described as

$$
l=\left\{l_{a}=1, \ldots, N\right\},
$$

where $l_{a}$ describes the number of neurons in the $a$-th layer.

The number of ANN inputs (in) depends on the number of $\left(\mathrm{u}_{\text {out }}^{\mathrm{i}}\right)$ samples and is equal $S_{u_{\text {out }}}$ and the number of ANN outputs (out) depends on the number of the specification parameters to be identified and is equal $I_{V C O}$.

For the following ANN, the number $s$ of $S_{u_{o u t}}$ was set to 20 and the number of $I_{V C O}$ was set to three. The presented artificial neural network has two hidden layers $\left(l_{1}, l_{2}\right)$ and the number of neurons in $l_{1}$ and $l_{2}$ is represented as:

$$
\begin{aligned}
& l_{1}=\frac{S_{u_{\text {out }}}}{2}, \\
& l_{2}=\frac{S_{u_{\text {out }}}}{4} .
\end{aligned}
$$

A bipolar sigmoid transfer function (tansig) [26] was used in all of the hidden layers. The presented artificial neural network was trained using the Levenberg-Marquardt algorithm and the mean square error was used as a performance function [26].

A data set of all of the $M_{c}$ is represented with those selected by the GA Sample that contained: $S A M=8000$ vectors, the training set Train contained $T R=5600$ vectors with the corresponding output set presented as Tout and the validation set (Valid) that contained the $V A L=2400$ vectors.

In the presented research, the genetic algorithm is used to provide the $S_{u_{\text {out }}}$ for the input of an ANN, which determines the values of $f x\left(u_{i n}^{i}\right), P_{d B m}^{i}$ and $P u l l^{i}$. The diagram of the GA and ANN connection is presented in (Fig. 7).

The evaluation of each individual took three steps:

1. Chromosome decoding (proposed indexes of 20 measurement samples of the VCO response).

2. Learning stage of the artificial neural network (ANN) using Train.

3. Verification stage using Valid Learning and the 
validation sets are disjoint.

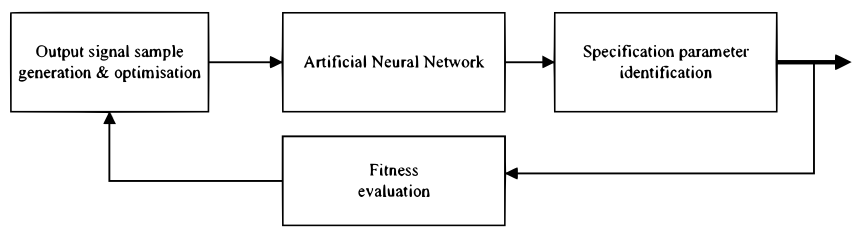

Fig. 7. Schematic of the optimisation and learning process.

Vector $S_{u_{\text {out }}}$, which was created with $w$ from the GA, permitted $p_{1}, p_{2}$ and $p_{3}$ to be designated by ANN. The fitness function is calculated based on the identify parameters. The result of $\left(F_{f}\right)$ is sent back to GA.

The fitness function $\left(F_{f}\right)$ of each individual on the basis of which the global minimum is calculated is as follows

$$
F_{f}=4 \times \frac{p_{1}}{6}+\frac{p_{2}}{0.5}+2 \times \frac{p_{3}}{12}
$$

where $p_{1}$ is a performance value of $f x\left(u_{i n}^{i}\right), p_{2}$ is a performance value of $P_{d B m}^{i}$ and $p_{3}$ is a performance value of Pull ${ }^{i}$.

\section{V.EXAMPLES}

The following section presents the results of the proposed method. All of the simulations were performed on a computer with the following parameters: Intel Core i5 $3.2 \mathrm{GHz}$ with $8 \mathrm{~GB}$ RAM.

In order to compare the results that were obtained from the use of the GA with ANN, all of the samples of the output signal were first given to the input of the neural network.

All of the tests presented below were compared with each other in terms of the efficiency of identifying the selected specification parameters of the VCO, but also in terms of the artificial neural network learning time and the artificial neural network response time for a given $S_{u_{\text {out }}}$.

\section{A. Case 1: All of the Samples of the Output Signal}

In the first case, all of the samples from the output $\left(S_{u_{\text {out }}}\right)$ signal were given to the input of the ANN. This solution was characterised by the longest training time $310 \mathrm{~s}$ and $40 \mathrm{~ms}$ response time but also had the highest efficiency of $p_{1}, p_{2}$ and $p_{3}$ (Table II).

TABLE II. THE RESULTS OF ALL OF THE $S_{u_{\text {out }}}$ THAT WERE OBTAINED FOR in OF THE ANN.

\begin{tabular}{|c|c|c|}
\hline $\boldsymbol{p}_{\mathbf{1}}[\boldsymbol{\%}]$ & $\boldsymbol{p}_{\mathbf{2}}[\boldsymbol{\%}]$ & $\boldsymbol{p}_{\mathbf{3}}[\boldsymbol{\%}]$ \\
\hline 4.8 & 5 & 9.1 \\
\hline \multicolumn{3}{|c|}{} \\
\hline \multicolumn{3}{|c|}{$\boldsymbol{S}_{\boldsymbol{u}_{\text {out }}}$} \\
\hline & $1 \ldots 256$ \\
\hline
\end{tabular}

The time required to get the last $S_{u_{\text {out }}}\left(T_{S_{\text {uout }}}\right)$ of the output signal was equal $5.67 \mathrm{~ns}$ for $u_{i n}^{1}$, equal $2.67 \mathrm{~ns}$ for $u_{i n}^{2}$, equal $1.96 \mathrm{~ns}$ for $u_{i n}^{3}$ and equal $1.73 \mathrm{~ns}$ for $u_{i n}^{4}$. The lowest error value was obtained after 33 epochs (Fig. 8).

Figure 9(a) presents a change in the direction of the mean square error and Fig. 9(b) a change in the mean square error in the function of the epoch number. As has been shown below (Fig. 8), the highest efficiency of identification was obtained after 33 epochs. The gradient of the mean square error value at 33 epoch was 0.16 and the mean square error reached a value of 0.00001 .

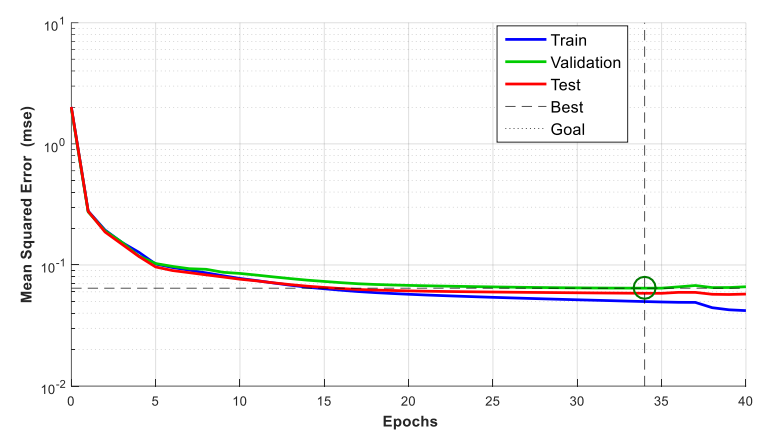

Fig. 8. Dependence of the Mean-Square Error (MSE) as a function of epoch for case 1 .
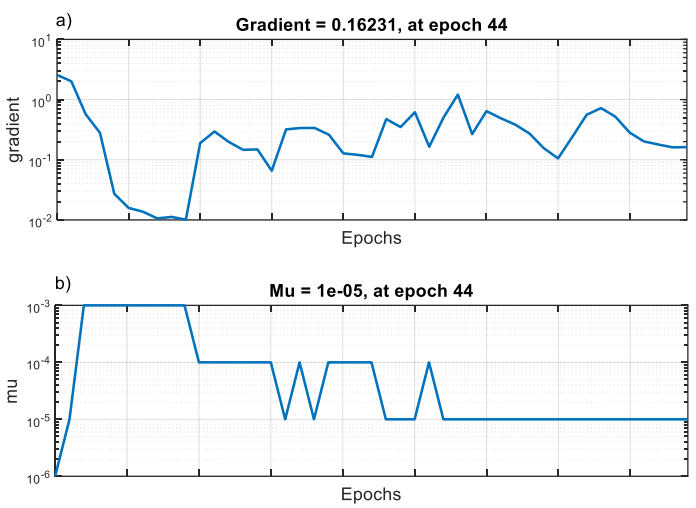

Fig. 9. Direction of the error change (a), and the change of the error value (b) in the case function for case 1 .

As can be seen in the Fig. 10, the largest differences in mapping appear for the $\boldsymbol{P}_{\boldsymbol{d B m}}$ parameter. The largest difference in identification occurred for the analyses contained in the $\boldsymbol{Y}_{\mathbf{6}}^{\text {mid }}$ deviation of the $W$ and $L$ parameters. The most accurate mapping in all of the deviations of $W$ and $L$ occurred for the $\boldsymbol{P} \boldsymbol{u l l}^{\boldsymbol{i}}$ parameter. The best mapping of all of the specification parameters was obtained for the deviation of the transistor's technological parameters by $Y_{2}^{\text {mid }}$.

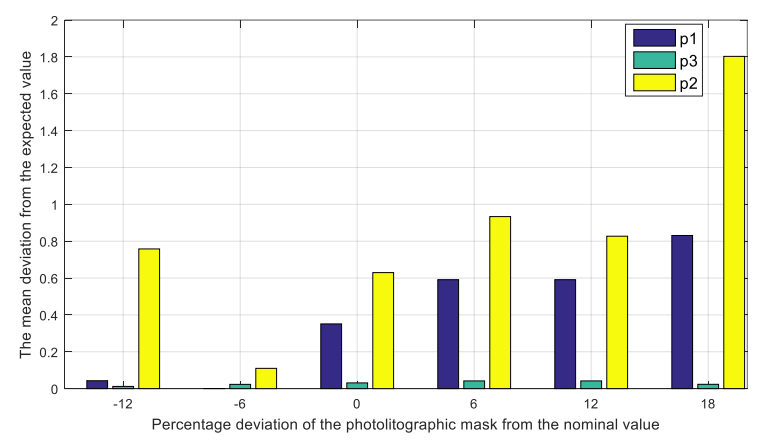

Fig. 10. Mean error value for the analysed values of the design specification as a function of the deviation of the photolithographic mask. Blue represents $\boldsymbol{p}_{\mathbf{1}}$, yellow represents $\boldsymbol{p}_{2}$ and green represents $\boldsymbol{p}_{3}$.

TABLE III. EXEMPLARY VALUES OF THE $\mathrm{P}_{1}$ SPECIFICATION: EXPECTED AND CALCULATED BY THE ANN.

\begin{tabular}{|c|c|c|}
\hline & $\begin{array}{c}\text { Expected Output } \\
{[\mathbf{G H z}]}\end{array}$ & ANN Output [GHz] \\
\hline $\boldsymbol{u}_{\boldsymbol{i n}}^{\mathbf{1}}$ & 1.381 & 1.331 \\
\hline $\boldsymbol{u}_{\boldsymbol{i n}}^{\mathbf{2}}$ & 0.387 & 0.370 \\
\hline $\boldsymbol{u}_{\boldsymbol{i n}}^{\mathbf{3}}$ & 2.583 & 2.465 \\
\hline $\boldsymbol{u}_{\boldsymbol{i n}}^{\mathbf{4}}$ & 3.009 & 2.876 \\
\hline
\end{tabular}

Table III presents the exemplary the values of $p_{1}$ that 
were calculated by the ANN compared with the expected values. For this example, the most accurate mapping occurred for $u_{i n}^{2}$, with a $0.017 \mathrm{GHz}$ error, the worst mapping occurred for $u_{i n}^{4}$, with a $0.132 \mathrm{GHz}$ error.

\section{B. Case 2: Samples Proposed in the Process of Minimising the Fitness Function $\left(F_{f}\right)$ Using the Genetic Algorithm}

In the second case, $S_{u_{\text {out }}}$ from the output signal that was proposed by the GA were given to the input of ANN. This solution was characterized by $17 \mathrm{~s}$ of training time and $14 \mathrm{~ms}$ of response time. The efficiency of $p_{1}, p_{2}$ and $p_{3}$ was about $0.5 \%$ less than in case 1 (Table IV).

TABLE IV. THE RESULTS THAT WERE OBTAINED FOR THE $S_{U_{\text {UUT }}}$ THAT WAS PROPOSED BY THE GA FOR THE in OF ANN.

\begin{tabular}{|c|c|c|}
\hline $\boldsymbol{p}_{\mathbf{1}}[\boldsymbol{\%}]$ & $\boldsymbol{p}_{\mathbf{2}}[\boldsymbol{\%}]$ & $\boldsymbol{p}_{\mathbf{3}}[\boldsymbol{\%}]$ \\
\hline 5.13 & 5.76 & 9.91 \\
\hline \multicolumn{3}{|c|}{$\boldsymbol{S}_{\boldsymbol{u}_{\text {out }}}$} \\
\hline 1571517273953636593111117155163177201211217231 \\
\hline
\end{tabular}

The time required to get the last $S_{u_{\text {out }}}\left(T_{S_{\text {uout }}}\right)$ of the output signal was equal to $5.36 \mathrm{~ns}$ for $u_{i n}^{1}$, was equal $2.42 \mathrm{~ns}$ for $u_{i n}^{2}$, was equal to $1.77 \mathrm{~ns}$ for $u_{i n}^{3}$ and was equal to $1.57 \mathrm{~ns}$ for $u_{i n}^{4}$. In this case, the lowest MSE value was reached after 106 epochs (Fig. 11).

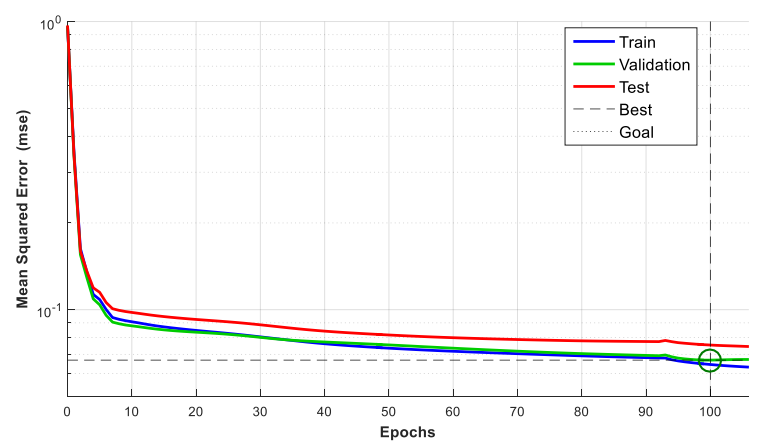

Fig. 11. Dependence of the Mean Square Error (MSE) in the function of Epoch for case 2.

The changing the direction of the mean square error (a) and changing mean square error in the function of the epoch number (b) are presented in the Fig. 12.
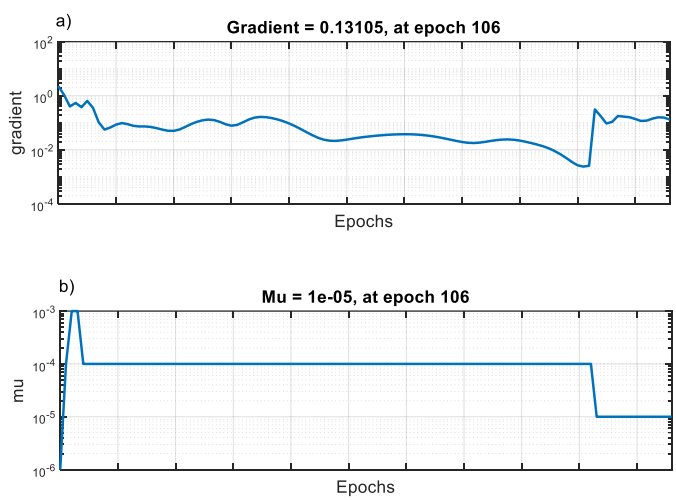

Fig. 12. Direction of error change (a) and change of the error value (b) in the case function for case 2 .

Figure 13 presents the mean mapping errors of the project specification parameter for the $\mathrm{S}_{\mathrm{u}_{\mathrm{out}}}$ that was proposed by the GA. As can be seen, the largest differences in the mapping for all of the $\mathrm{Y}^{\mathrm{mid}}$ occur for $\mathrm{P}_{\mathrm{dBm}}$.

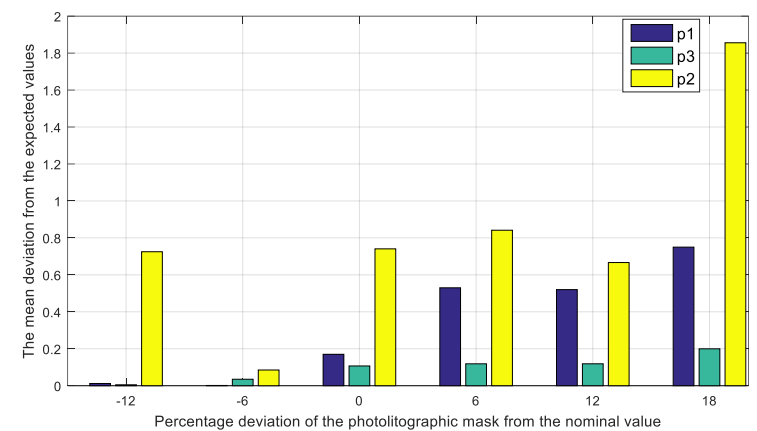

Fig. 13. Mean error value for the analysed values of the design specification as a function of the photolithographic mask deviation. Blue represents $\boldsymbol{p}_{\mathbf{1}}$, yellow represents $\boldsymbol{p}_{\mathbf{2}}$ and green represents $\boldsymbol{p}_{\mathbf{3}}$.

As was shown at Fig. 11, the highest efficiency of identification was obtained after 106 epochs. The gradient of the mean square error value at 106 epochs was 0.13 and the mean square error reached a value of 0.00001 .

The most accurate mapping for all of the deviations $W$ and $L$ occurred for parameter $f x\left(u_{i n}^{i}\right)$. The best mapping of all of the specification parameters was obtained by $\boldsymbol{Y}_{2}^{\text {mid }}$ for the deviation of the transistor's technological parameters.

TABLE V. EXEMPLARY VALUES OF THE $\mathrm{P}_{1}$ SPECIFICATION: EXPECTED AND CALCULATED BY THE ANN.

\begin{tabular}{|c|c|c|}
\hline & $\begin{array}{c}\text { Expected Output } \\
\text { [GHz] }\end{array}$ & ANN Output [GHz] \\
\hline $\boldsymbol{u}_{\boldsymbol{i n}}^{\mathbf{1}}$ & 1.381 & 1.202 \\
\hline $\boldsymbol{u}_{\boldsymbol{i n}}^{\mathbf{2}}$ & 0.387 & 0.373 \\
\hline $\boldsymbol{u}_{\boldsymbol{i n}}^{\mathbf{3}}$ & 2.583 & 2.434 \\
\hline $\boldsymbol{u}_{\boldsymbol{i n}}^{\mathbf{4}}$ & 3.009 & 2.818 \\
\hline
\end{tabular}

Table $\mathrm{V}$ presents the exemplary values of $\mathrm{p}_{1}$ that were calculated by the ANN compared with the desired values. For this example, the most accurate mapping occurred for $u_{i n}^{2}$, with a $0.014 \mathrm{GHz}$ error, the worst mapping occurred for $u_{i n}^{4}$, with a $0.191 \mathrm{GHz}$ error.

\section{Case 3: Random $S_{u_{\text {out }}}$ Selection}

In case $3, S_{u_{\text {out }}}$ equal to 20 random samples of $u_{\text {out }}$ were given to the input of the ANN. This solution was characterised by $23 \mathrm{~s}$ of training time and $15 \mathrm{~ms}$ of response time. The efficiency of $p_{1}$ and $p_{3}$ are presented in Table VI.

TABLE VI. THE RESULTS OBTAINED FOR $\mathrm{S}_{\mathrm{U}_{\text {out }}}$ EQUAL TO 20 RANDOM SAMPLES OF U OUT FOR THE IN OF THE ANN

\begin{tabular}{|c|c|c|}
\hline $\boldsymbol{p}_{\mathbf{1}}[\boldsymbol{\%}]$ & $\boldsymbol{p}_{\mathbf{2}}[\boldsymbol{\%}]$ & $\boldsymbol{p}_{\mathbf{3}}[\boldsymbol{\%}]$ \\
\hline 6.87 & 6.1 & 0.12 \\
\hline \multicolumn{3}{|c|}{$\boldsymbol{S}_{\boldsymbol{u}_{\text {out }}}$} \\
\hline 284957777981120125166174185186187207225226227230 \\
\hline \multicolumn{3}{|c|}{231239} \\
\hline
\end{tabular}

The time required to get the last $S_{u_{\text {out }}}\left(T_{S_{\text {uout }}}\right)$ of the output signal was equal to $5.59 \mathrm{~ns}$ for $u_{i n}^{1}$, was equal to $2.45 \mathrm{~ns}$ for $u_{i n}^{2}$, was equal to $1.8 \mathrm{~ns}$ for $u_{i n}^{3}$ and was equal to $1.6 \mathrm{~ns}$ for $u_{i n}^{4}$. In this case, the lowest MSE value was reached after 346 epochs (Fig. 14).

As can be seen in Fig. 13, the highest efficiency of identification was obtained after 346 epochs.

The gradient of the mean square error value at epoch 346 was 0.23 and the mean square error reached a value of 0.00001 (Fig. 15). 


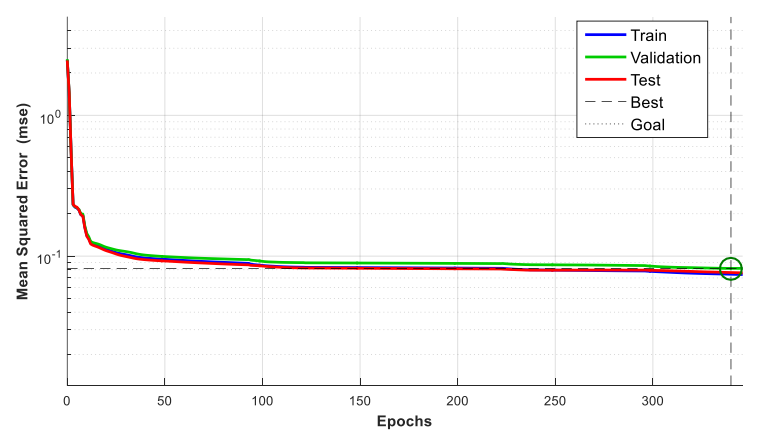

Fig. 14. Dependence of the Mean Square Error (MSE) in the function of Epoch for case 3.
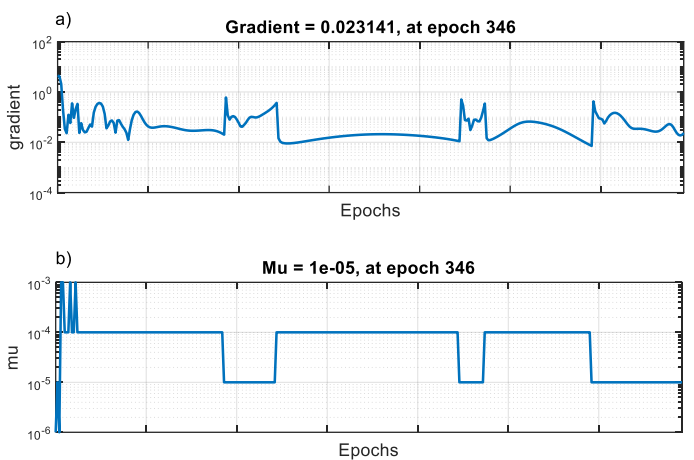

Fig. 15. Direction of the error change (a) and change in the error value (b) in the case function for case 3 .

TABLE VII. EXEMPLARY VALUES OF THE $P_{2}$ SPECIFICATION: EXPECTED AND CALCULATED BY THE ANN

\begin{tabular}{|c|c|c|}
\hline & $\begin{array}{c}\text { Expected Output } \\
{[\mathbf{d B m}]}\end{array}$ & ANN Output [dBm] \\
\hline $\boldsymbol{u}_{\boldsymbol{i n}}^{\mathbf{1}}$ & 1.400 & 1.387 \\
\hline $\boldsymbol{u}_{\boldsymbol{i n}}^{\mathbf{2}}$ & 1.447 & 1.558 \\
\hline $\boldsymbol{u}_{\boldsymbol{i n}}^{\mathbf{3}}$ & 1.463 & 1.535 \\
\hline $\boldsymbol{u}_{\boldsymbol{i n}}^{\mathbf{4}}$ & 1.487 & 1.586 \\
\hline
\end{tabular}

Table VII presents the exemplary values of $\mathrm{p}_{2}$ as calculated by the ANN compared with the desired values. For this example, the most accurate mapping occurred for $u_{i n}^{1}$, with a $0.013 \mathrm{dBm}$ error, the worst mapping occurred for $u_{i n}^{2}$, with a $0.111 \mathrm{dBm}$ error.

\section{Case 4: Samples Proposed in the Process of Minimizing} the Modified Fitness Function $\left(F_{f}\right)$ Using the Genetic Algorithm

In this case, the fitness function $\left(F_{f}\right)$ was modified. The aim of the modification of the fitness function was to add an additional parameter that would allow the promotion of those solutions whose index of the last $S_{u_{\text {out }}}$ was the earliest. The new $F_{f}$ is presented below

$$
F_{f}=4 \times \frac{p_{1}}{6}+\frac{p_{2}}{0.5}+2 \times \frac{p_{3}}{12}-\frac{0.04}{L i} .
$$

where $L i$ is the last index of the $S_{u_{\text {out }}}$.

In case 4 , the $S_{u_{o u t}}$ from the output signal that was proposed by the GA with the modified $F_{f}$ were given to the input of the ANN. This solution was characterised by $10 \mathrm{~s}$ of training time and $13.4 \mathrm{~ms}$ of response time. The efficiency of $p_{1}, p_{2}$ and $p_{3}$ about $0.5 \%$ less than in case 1 (Table VIII).

The time required to get the last $S_{u_{\text {out }}}\left(T_{S_{\text {uout }}}\right)$ of the output signal was equal to $5.2 \mathrm{~ns}$ for $u_{i n}^{1}$, was equal to $2.2 \mathrm{~ns}$ for $u_{i n}^{2}$, was equal $1.6 \mathrm{~ns}$ for $u_{i n}^{3}$ and was equal to $1.42 \mathrm{~ns}$ for $u_{\text {in }}^{4}$.

In this case, the lowest MSE value was reached after 157 epochs (Fig. 17).

As can be seen in Fig. 16, the highest efficiency of identification was obtained after 157 epochs. The gradient of the mean square error value at epoch 157 was 0.065 and the mean square error reached a value of 0.000001 .

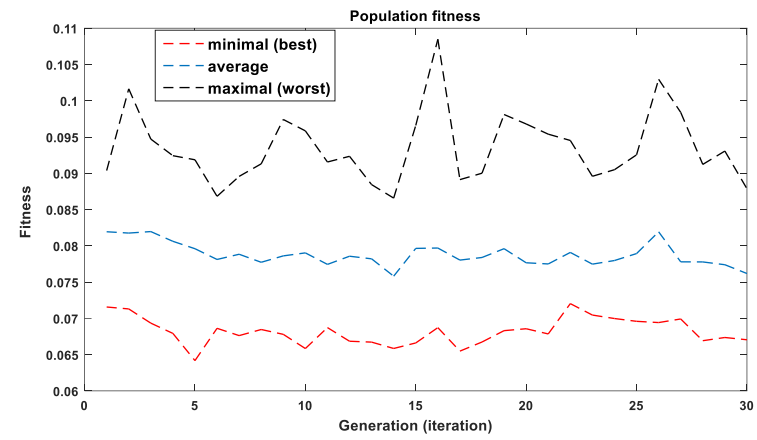

Fig. 16. Fitness progress of the GA evolution after modified $F_{f}$.

TABLE VIII. THE RESULTS OBTAINED FOR THE $S_{U_{\text {OUT }}}$ THAT WAS PROPOSED BY THE GA WITH THE NEW $F_{F}$ FOR IN OF THE ANN.

\begin{tabular}{|c|c|c|}
\hline $\boldsymbol{p}_{\mathbf{1}}[\boldsymbol{\%}]$ & $\boldsymbol{p}_{\mathbf{2}}[\boldsymbol{\%}]$ & $\boldsymbol{p}_{\mathbf{3}}[\boldsymbol{\%}]$ \\
\hline 5.6 & 2.9 & 10.5 \\
\hline \multicolumn{3}{|c|}{$\boldsymbol{S}_{\boldsymbol{u}_{\text {out }}}$} \\
\hline 131339435361677375798385117119131155159210213 \\
\hline
\end{tabular}

Figure 18 shows the mean deviations from the identification of the analysed specification parameters of the CUT design specification. The maximum deviation was obtained for $Y_{\mathbf{6}}^{\text {mid }}$ for $\boldsymbol{P}_{\boldsymbol{d B m}}$.

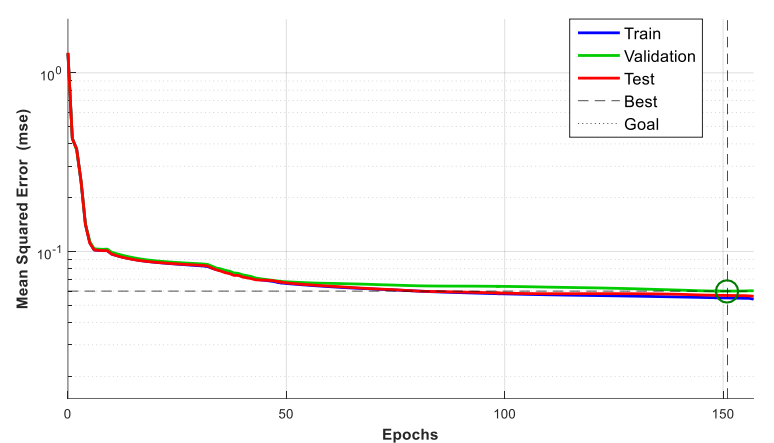

Fig. 17. Dependence of the Mean Square Error (MSE) in the function of Epoch for case 4.

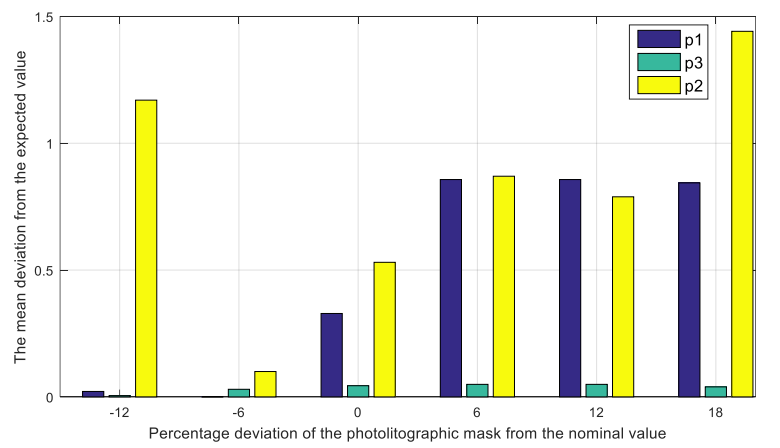

Fig. 18. Mean error value for the analysed values of the design specification as a function of the photolithographic mask deviation. Blue represents $\boldsymbol{p}_{\mathbf{1}}$, yellow represents $\boldsymbol{p}_{\mathbf{2}}$ and green represents $\boldsymbol{p}_{3}$.

Table IX presents the exemplary values of $\mathrm{p}_{3}$ that were 
calculated by the ANN compared with the desired values. For this example, the most accurate mapping occurred for $u_{i n}^{4}$, with a $0.11 \mathrm{dBr}$ error, the worst mapping occurred for $u_{\text {in }}^{1}$ with a $8.01 \mathrm{dBr}$ error.

TABLE IX. EXEMPLARY VALUES OF THE $\mathrm{P}_{3}$ SPECIFICATION: EXPECTED AND CALCULATED BY THE ANN.

\begin{tabular}{|c|c|c|}
\hline & $\begin{array}{c}\text { Expected Output } \\
{[\mathbf{d B r}]}\end{array}$ & ANN Output [dBr] \\
\hline $\boldsymbol{u}_{\boldsymbol{i n}}^{\mathbf{1}}$ & -96.9 & -88.89 \\
\hline $\boldsymbol{u}_{\boldsymbol{i n}}^{\mathbf{2}}$ & -110.5 & -108.55 \\
\hline $\boldsymbol{u}_{\boldsymbol{i n}}^{\mathbf{3}}$ & -93.9 & -94.17 \\
\hline $\boldsymbol{u}_{\boldsymbol{i n}}^{\mathbf{4}}$ & -107.9 & -108.01 \\
\hline
\end{tabular}

\section{CONCLUSIONS}

The presented paper discusses the problem of reducing the identification time of the selected specification parameters of a Voltage-Controlled Oscillator in its ring oscillator structure. The use of the genetic algorithm together with an artificial neural network allowed selected specification parameters of the CUT to be identified based on the proposed samples that were obtained from the CUT response signal. The proposed method permitted the test time to be significantly reduced (see Sec. V), the total time $\left(T_{\text {overall }}=T_{\text {resp }}+T_{S_{\text {uout }}}\right)$ required to obtain the $p_{1}, p_{2}$ and $p_{3}$ values for the analysed system response for $S_{u_{\text {out }}}=256$ was equal ca. $40 \mathrm{~ms}$, where for the $S_{u_{\text {out }}}$ that was proposed by the GA (case 4), it was equal ca. $13.4 \mathrm{~ms}$. Moreover, in the proposed method, the mapping errors are slightly higher than was in case $1, \Delta p_{1}=0.0077, \Delta p_{2}=0.024$ and $\Delta p_{3}=0.0134$. The method, which is based on the application of a GA with the ANN, also has one more significant advantage, it allows the time for the stimulation of the circuit to be reduced as it requires the acquisition of fewer samples than for the full system response being considered. The proposed method is simple and fast to implement, because it does not require complicated calculations. It should also be mentioned that the proposed method is sensitive because the fitness function can radically change the efficiency of the entire process. Due to its simplicity, it can be used to identify other or more parameters of the CUT design specification.

\section{REFERENCES}

[1] M. Tadeusiewicz, S. Halgas, "Diagnosis of soft spot short defects in analog circuits considering the thermal behaviour of the chip", Metrology and Measurement Systems, vol. 23, no. 2, pp. 239-250, 2016. DOI: 10.1515/mms-2016-0023.

[2] B. Long, M. Li, H. Wang, S. Tian, "Diagnostics of analog circuits based on LS-SVM using time-domain features", Circuits Syst. Signal Process., vol. 32, no. 6, pp. 2683-2706, 2013. DOI: 10.1007/s00034013-9614-3.

[3] S. F. Wang, "Low-voltage, full-swing voltage-controlled oscillator with symmetrical even-phase outputs based on single-ended delay cells", IEEE Trans. Very Large Scale Integr. VLSI Syst., vol. 23, no. 9, pp. 1801-1807, 2015. DOI: 10.1109/TVLSI.2014.2345277.

[4] L. Chruszczyk, "Automatic test bench for selected transmission parameters of power line conductors", Int. J. Electron. Telecomm., vol. 61, no. 1, pp. 59-65, 2015. DOI: 10.1515/eletel-2015-0008.

[5] P. Jantos, T. Golonek, J. Rutkowski, "An analogue electronic circuits specification driven testing with the use of time domain response's features", in Proc. 18th Int. Conf. Mixed Design of Integrated Circuits and Systems (MIXDES 2011), 2011, Gliwice, Poland, pp. 485-489.

[6] K. Huang, H. G. Stratigopoulos, S. Mir, C. Hora, Y. Xing, B. Kruseman, "Diagnosis of local spot defects in analog circuits",
IEEE Trans. Instrum. Meas., vol. 61, no. 10, pp. 2701-2712, 2012 DOI: 10.1109/TIM.2012.2196390.

[7] D. Grzechca, "Construction of an expert system based on fuzzy logic for diagnosis of analog electronic circuits", Int. J. Electron. Telecommun., vol. 61, no. 1, pp. 77-82, 2015. DOI: 10.1515/eletel2015-0010.

[8] P. Kabisatpathy, A. Barua, S. Sinha, Fault Diagnosis of Analog Integrated Circuits, Berlin/Heidelberg: Springer-Verlag, 2005. DOI: 10.1007/b135977.

[9] A. S. Orgenci, G. Dundar, S. Balkur, "Fault-tolerant training of neural networks in the presence of MOS transistor mismatches", IEEE Trans. Circuits and Systems II: Analog and Digital Signal Processing, vol. 48, no. 3, pp. 272-281, 2001. DOI: 10.1109/82.924069.

[10] A. Robotycki, R. Zielonko, "Fault diagnosis of analog piecewise linear circuits based on homotopy", IEEE Trans. Instrum. Meas., vol. 51, no. 4, pp. 876-881, 2002. DOI: 10.1109/TIM.2002.803515.

[11] M. Tadeusiewicz, S. Halgas, "Global and local parametric diagnosis of analog short-channel CMOS circuits using homotopy-simplicial algorithm", Int. J. Circuit Theory Appl., vol. 42, no. 10, pp. 10511068, 2014. DOI: $10.1002 /$ cta.1904.

[12] S. Temich, D. Grzechca, K. Tokarz, "Application of fuzzy set expert system for testing selected specification parameters of voltagecontrolled oscillator", Adv. Intell. Syst. Comput., vol. 659, pp. 330340, 2018. DOI: 10.1007/978-3-319-67792-7_33.

[13] H. G. Stratigopoulos, S. Mir, "Analog test metrics estimates with PPM accuracy", IEEE/ACM Int. Conf. Computer-Aided Design (ICCAD 2010), San Jose, CA, USA, 2010, pp. 241-247. DOI: 10.1109/ICCAD.2010.5654165.

[14] M. Radfar, M. M. Green, "An LC voltage-controlled oscillator with supply sensitivity compensation method", IEEE 60th Int. Midwest Symposium on Circuits and Systems (MWSCAS 2017), Boston, MA, USA, 2017, pp. 799-802. DOI: 10.1109/MWSCAS.2017.8053044.

[15] K. Huang, H. G. Stratigopoulos, S. Mir, "Bayesian fault diagnosis of RF circuits using nonparametric density estimation", 19th IEEE Asian Test Symposium, Shanghai, China, 2010, pp. 295-298. DOI: 10.1109/ATS.2010.57.

[16] H. Koo, C. Y. Kim, S. Hong, "Design and analysis of $239 \mathrm{GHz}$ CMOS Push-Push transformer-based VCO with high efficiency and wide tuning range", IEEE Trans. Circuits and Systems I: Regular Papers, vol. 62, no. 7, pp. 1883-1893, 2015. DOI: 10.1109/TCSI.2015.2426957.

[17] F. Aminian, M. Aminian, "Fault diagnosis of analog circuits using Bayesian neural networks with wavelet transform as preprocessor", Journal of Electronic Testing, vol. 17, no. 1, pp. 29-36, 2001. DOI: 10.1023/A:1011141724916.

[18] S. Temich, D. Grzechca, "Application of neural network for testing voltage-controlled oscillators”, IFAC-PapersOnLine, vol. 49, no. 25, pp. 407-412, 2016. DOI: 10.1016/j.ifacol.2016.12.081.

[19] S. C. Gladson, "A 100/10-MHz injection controlled LC-VCO", in Int. Conf. Circuit, Power and Computing Technologies (ICCPCT 2016), Nagercoil, India, 2016, pp. 1-4. DOI: 10.1109/ICCPCT.2016.7530171.

[20] M. Burbidge, A. Richardson, "Phase-locked loop test methodologies: Current characterization and production test practices," in Test and Diagnosis of Analogue, Mixed-Signal and RF Integrated Circuits: the system on chip approach, UK: IET, pp. 277-308. DOI: 10.1049/pbcs019e_ch9.

[21] Ulrich L. Rohde, Microwave and Wireless Synthesizers: Theory and Design, Wiley, 1997, p. 656.

[22] I. Toihria, R. Ayadi, M. Masmoudi, "High performance BIST PLL approach for VCO testing", in 1st Int. Conf. Advanced Technologies for Signal and Image Processing (ATSIP 2014), Sousse, Tunisia, 2014, pp. 517-522. DOI: 10.1109/ATSIP.2014.6834669.

[23] C. L. Singh, A. J. Gogoi, C. Anandini, K. L. Baishnab, "Low-noise CMOS differential-amplifier design using automated-design methodology", in Devices for Integrated Circuit (DevIC 2017), Kalyani, India, 2017, pp. 326-330. DOI: 10.1109/DEVIC.2017.8073962.

[24] W. Meyer, H. T. Vierhaus, "Switch-level fault simulation for nontrivial faults based on abstract data types", in Proc. Advanced Computer Technology, Reliable Systems and Applications, Bologna, Italy, 1991, pp. 233-237. DOI: 10.1109/CMPEUR.1991.257388.

[25] Z. Liu, S. K. Chaganti, D. Chen, "Improving time-efficiency of faultcoverage simulation for MOS analog circuit", IEEE Trans. Circuits and Systems I: Regular Papers, vol. 65, no. 5, pp. 1664-1674, 2017. DOI: 10.1109/TCSI.2017.2751561.

[26] J. Leski, Systemy neuronowo-rozmyte. Warszawa: Wydawnictwa Naukowo-Techniczne, 2008. (in Polish). 